\title{
Mycena genomes resolve the evolution of fungal bioluminescence
}

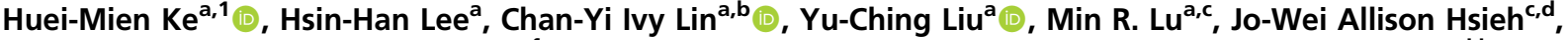

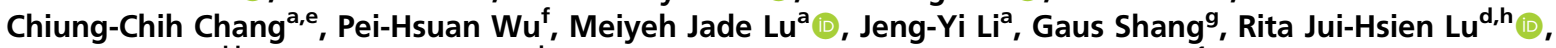

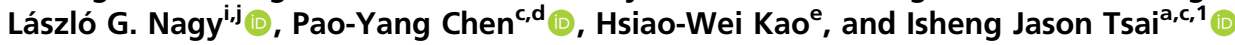

${ }^{a}$ Biodiversity Research Center, Academia Sinica, Taipei 115, Taiwan; bepartment of Molecular, Cellular and Developmental Biology, Yale University, New Haven, CT 06520; 'Genome and Systems Biology Degree Program, Academia Sinica and National Taiwan University, Taipei 106, Taiwan; ${ }^{\mathrm{d}}$ Institute of Plant and Microbial Biology, Academia Sinica, Taipei 115, Taiwan; ${ }^{\mathrm{E} D e p a r t m e n t}$ of Life Sciences, National Chung Hsing University, Taichung 402, Taiwan; ${ }^{\mathrm{f}}$ Master Program for Plant Medicine and Good Agricultural Practice, National Chung Hsing University, Taichung 402, Taiwan; ${ }^{9}$ Department of Biotechnology, Ming Chuan University, Taoyuan 333, Taiwan; ${ }^{h}$ Department of Medicine, Washington University in St. Louis, St. Louis, MO 63110; 'Synthetic and Systems Biology Unit, Biological Research Centre, 6726 Szeged, Hungary; and 'jDepartment of Plant Anatomy, Institute of Biology, Eötvös Loránd University, Budapest, 1117 Hungary

Edited by Manyuan Long, University of Chicago, Chicago, IL, and accepted by Editorial Board Member W. F. Doolittle October 28, 2020 (received for review May 27, 2020)

Mushroom-forming fungi in the order Agaricales represent an independent origin of bioluminescence in the tree of life; yet the diversity, evolutionary history, and timing of the origin of fungal luciferases remain elusive. We sequenced the genomes and transcriptomes of five bonnet mushroom species (Mycena spp.), a diverse lineage comprising the majority of bioluminescent fungi. Two species with haploid genome assemblies $\sim 150 \mathrm{Mb}$ are among the largest in Agaricales, and we found that a variety of repeats between Mycena species were differentially mediated by DNA methylation. We show that bioluminescence evolved in the last common ancestor of mycenoid and the marasmioid clade of Agaricales and was maintained through at least $\mathbf{1 6 0}$ million years of evolution. Analyses of synteny across genomes of bioluminescent species resolved how the luciferase cluster was derived by duplication and translocation, frequently rearranged and lost in most Mycena species, but conserved in the Armillaria lineage. Luciferase cluster members were coexpressed across developmental stages, with the highest expression in fruiting body caps and stipes, suggesting fruiting-related adaptive functions. Our results contribute to understanding a de novo origin of bioluminescence and the corresponding gene cluster in a diverse group of enigmatic fungal species.

fungal bioluminescence | luciferase cluster evolution | genome evolution

$T^{1}$ he genus Mycena comprises 600 small mushroom species widely distributed around the world (1). Also known as bonnet mushrooms, Mycena species are usually characterized by a bell-shaped cap, a thin stem (Fig. $1 A$ ), and a gilled or porioid hymenophore (2). Mycena also have a diversity of life history strategies; while many species are saprotrophic, they can be pathogenic as well as mycorrhizal (3). Despite its vast diversity of lifestyles and phenotypes, there are many questions concerning the basic biology, ecology, and genomics of this genus. One particular fascinating trait is bioluminescence, which is most widespread in the genus Mycena. At least 68 of the 81 known bioluminescent fungi belong to Mycena (4), yet these account for less than $12 \%$ of the approximately 600 Mycena species (5), suggesting an intricate loss/gain history and potential convergence within the genus.

Fungal light emission involves two main steps. First, a luciferin precursor of hispidin is hydroxylated by hispidin-3-hydroxylase $(\mathrm{H} 3 \mathrm{H})$ into 3-hydroxyhispidin (luciferin) (6). Oxygen is then added to the luciferin by luciferase, producing a high-energy intermediate which is subsequently decomposed, yielding light emission. Previously, Kotlobay et al. have identified the fungal luciferase, which is physically adjacent to these enzymes and forms a gene cluster containing luciferase, hispidin synthase, and $\mathrm{H} 3 \mathrm{H}$ (7). This cluster was found to be conserved across bioluminescent fungi of three lineages: Armillaria, mycenoid, and Omphalotus (7). Phylogeny reconstruction suggested that luciferase originated in early Agaricales. Armillaria and Omphalotus belong to the marasmioid clade, whereas Mycena was recently found to be sister of the marasmioid clade (8). Recent genome sequencing efforts in the marasmioid clade revealed diverse genomic and life history traits, including genome expansion and pathogenicity in Armillaria spp. (9), novel wood decay strategies (10), or fruiting body development (11). Genomes of two Mycena species were sequenced (7); however, the fragmented assemblies (N50 5.8 to $16.7 \mathrm{~kb}$ ) impeded comparative genomic analyses of features such as synteny (12). These resources provide a substrate for studies of genome evolution and of bioluminescence in fungi; however, several key questions remain unresolved. Here, we set out to

\section{Significance}

We present the genomes of five new bonnet mushroom Mycena species, formerly the last fungal bioluminescent lineage lacking reference genomes. These genome-scale datasets allowed us to construct an evolutionary model pinpointing all possible changes in the luciferase cluster across all fungi and additional genes involved in bioluminescence. We show that luciferase clusters were differentially lost in different fungal lineages and in particular a substantial loss was observed in the mycenoid lineage. This can be attributed to genome regions of Mycena that underwent different evolutionary dynamics. Our findings offer insights into the evolution of how a gene cluster emerged 160 million years ago and was frequently lost or maintained due to differences in genome plasticity.

Author contributions: H.-M.K. and I.J.T. conceived the study; I.J.T. led the study; H.-M.K. C.C.-C., G.S., and H.-W.K. sampled the Mycena species in Taiwan; H.-M.K., P.-H.W., and C.-Y.I.L. conducted the experiments; M.J.L. and J.-Y.L. designed the Illumina sequencing experiment; H.-H.L. and I.J.T. performed the assemblies and annotations of the Mycena genomes; H.-M.K., H.-H.L., Y.-C.L., and I.J.T. conducted the repeat analysis; L.G.N. and I.J.T. carried out phylogenomics analyses and the divergence time estimation; H.-M.K., H.-H.L., Y.-C.L., and I.J.T. carried out comparative genomic analyses; H.-M.K. and M.R.L. analyzed the RNA-seq data; H.-M.K., H.-H.L., J.-W.A.H., R.J.-H.L., and P.-Y.C., carried out the methylation analyses; and H.-M.K. and I.J.T. wrote the manuscript with input from L.G.N. and P.-Y.C.

The authors declare no competing interest.

This article is a PNAS Direct Submission. M.L. is a guest editor invited by the Editorial Board.

This open access article is distributed under Creative Commons Attribution-NonCommercialNoDerivatives License 4.0 (CC BY-NC-ND).

${ }^{1}$ To whom correspondence may be addressed. Email: cotton@sinica.edu.tw or ijtsai@ sinica.edu.tw.

This article contains supporting information online at https://www.pnas.org/lookup/suppl/ doi:10.1073/pnas.2010761117/-/DCSupplemental.

First published November 23, 2020. 


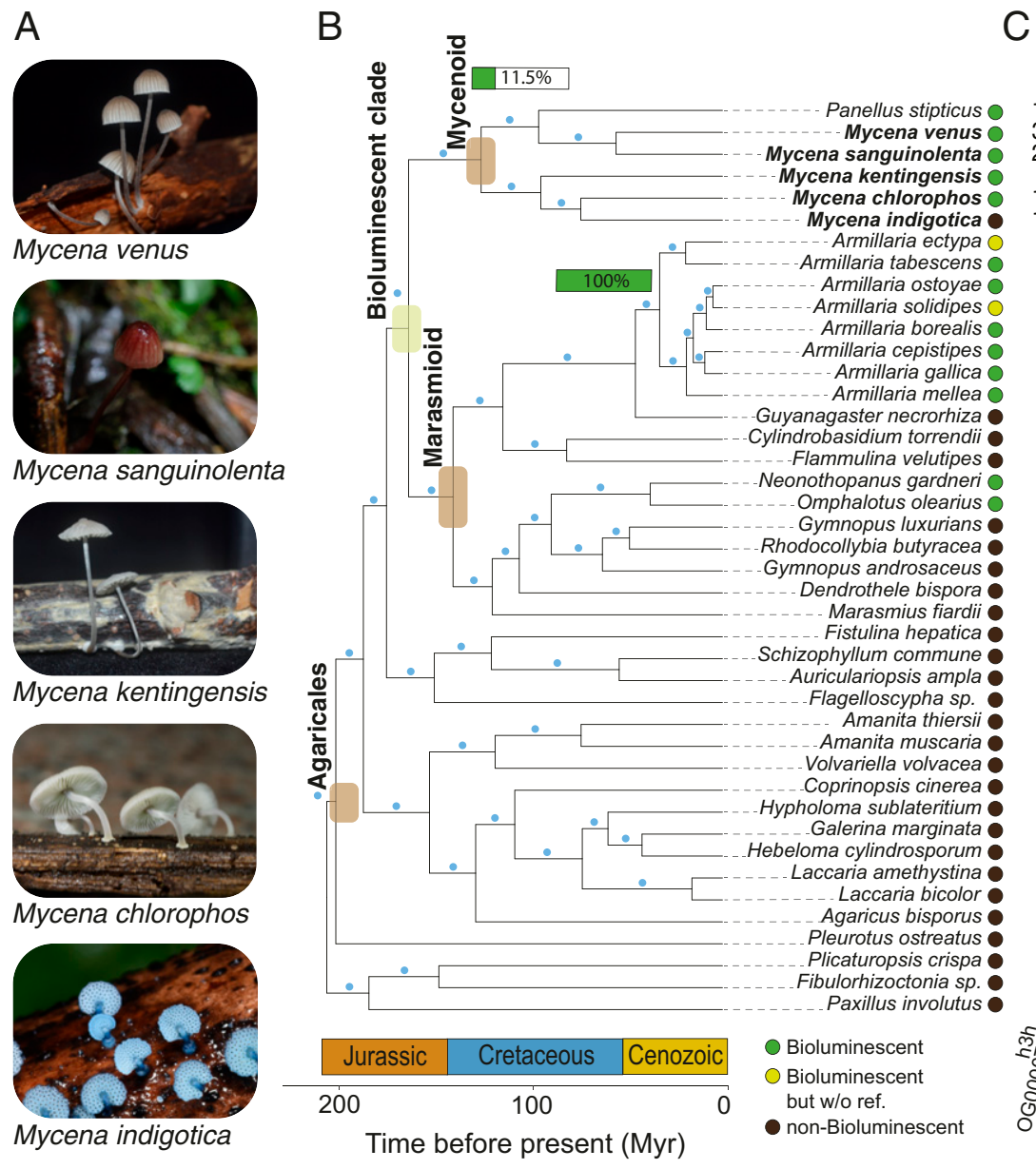

C $\mathrm{D}$

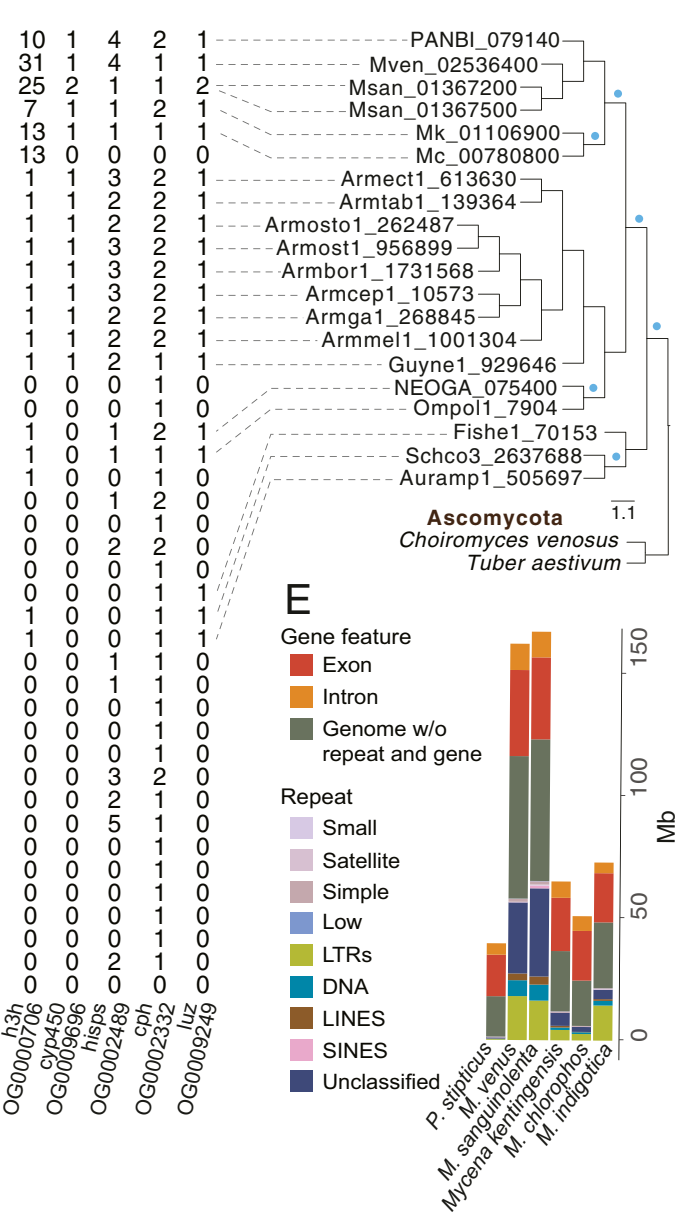

Fig. 1. Phylogenomic analysis of Mycena and related fungi. (A) The five species sequenced in this study. (B) Species trees inferred from a concatenated supermatrix of the gene alignments using the 360 single-copy orthogroups. The $x$ axis denotes divergence time estimates. Blue dot on a branch indicates a bootstrap value $>90$. Green horizontal bars indicate the percentage of bioluminescent fungi found in either the mycenoid or the Armillaria lineage. (C) Gene copy number in the OGs associated with luciferin biosynthesis pathway including luciferase (luz), hispidin-3-hydroxylase ( $h 3 h$ ), hispidin synthase (hisps), cytochrome P450 (cyp450), and caffeylpyruvate hydrolase ( $c p h)$. (D) Reconciliated phylogeny of fungal luciferase. Blue dot on a branch indicates a bootstrap value $>90$. $(E)$ Haploid genome sizes for mycenoid species broken down by repeat types and gene features. Repeats including TEs are LTRs, long interspersed nuclear elements (LINES), short interspersed nuclear elements (SINEs), DNA transposons (DNA), and other types of repeats, such as small RNA (small), simple repeats (simple), and low complexity repeats (low).

understand how the luciferase cluster originated and was lost or retained, determine levels of variation in this cluster across these lineages, and identify novel genes involved in bioluminescence.

To gain insights into the evolution of fungal bioluminescence and the ecology of mycenoid species, we sequenced the genomes of four bioluminescent (Mycena chlorophos, Mycena kentingensis, Mycena sanguinolenta, and Mycena venus) and one nonbioluminescent (Mycena indigotica) species. We conducted comparative genomics with representative genomes of all bioluminescent fungal clades, putting particular emphasis on genomewide synteny to investigate the evolutionary dynamics of the luciferase gene cluster through hundreds of millions of years. The variability in genome sizes among Mycena is likely associated with the differential expansion of repeats in the genomes, potentially due to the differential control on repeat activity by DNA methylation. Members of the luciferase cluster were up-regulated in the transcriptome of bioluminescent mycelium, and coexpression analyses identified specific genes likely to associate with the bioluminescence and development in Mycena. Based on comparative analyses from 15 available genomes of bioluminescent fungi, we reconstructed and formulated a model for the evolution of fungal bioluminescence.

\section{Results}

Assemblies and Annotations of Five Mycena Species. We sequenced the genomes of the bioluminescent fungi M. chlorophos, M. kentingensis, $M$. sanguinolenta, and $M$. venus, as well as the nonbioluminescent $M$. indigotica (Fig. $1 A$ ). These species were chosen for their phylogenetic positions (SI Appendix, Fig. S1) and because they displayed different bioluminescence intensities. An initial assembly of each species was produced from Oxford Nanopore reads of each species (SI Appendix, Table S1) using the Canu (13) assembler. Only $M$. indigotica was successfully isolated from a basidiospore, and the four species that were isolated from heterokaryotic mycelium yielded assemblies 1.3 to 1.8 times larger than haploid genome sizes estimated from Illumina reads using GenomeScope (14) (SI Appendix, Table S2). Mitochondrial genomes in these species were separately assembled into single circular contigs of 88.3 to $133 \mathrm{~kb}$ long (SI Appendix, Fig. S2), and haploid nuclear genomes were constructed. The assemblies were 
further polished using Illumina reads and had consensus quality values (QVs) of 31.1 to 36.8 (SI Appendix, Table S2), which is similar to the QVs of recently published nanopore assemblies in human (also polished with Illumina reads; 23.7 to 43.5) (15) These haploid nuclear genomes were 50.9 to $167.2 \mathrm{Mb}$ long, and two of them were among the largest in Agaricales reported to date. The assemblies consisted of 30 to 155 contigs with N50 2.0 to $5.5 \mathrm{Mb}$ (SI Appendix, Table S2), which were comparable to representative fungal reference assemblies (SI Appendix, Fig. S3) and allowed for synteny comparisons (12). Stretches of TTAGGG hexamers were identified at the end of scaffolds, indicating telomeric repeats commonly found in Agaricales $(16,17)$. The largest scaffolds in $M$. indigotica and $M$. kentingensis were telomere to telomere, indicating gapless chromosomes.

Using a combination of reference fungal protein homology support and mycelium transcriptome sequencing (Dataset S1), 13,940 to 26,334 protein-encoding genes were predicted in the Mycena genomes using MAKER2 (18) pipeline, and were 92.1 to 95.3\% complete (SI Appendix, Table S3) based on BUSCO (19) analysis. Orthology inference using Orthofinder $(20,21)$ placed these gene models and those of 37 other Basidiomycota genomes (SI Appendix, Table S4) into 22,244 orthologous groups (OGs; SI Appendix, Table S5). Of these OGs, $44.3 \%$ contained at least one ortholog from another basidiomycete, while 15 to $29 \%$ of the proteomes in each Mycena species were species specific (Dataset $\mathrm{S} 2$ ). The genome sizes were positively correlated with proteome sizes, with the largest ( $M$. sanguinolenta) and smallest $(M$. chlorophos) varying two- and threefold, respectively. Interestingly, the mitochondrial genomes were larger in species with smaller genomes, and this was because nine out of 16 genes had gained many introns (SI Appendix, Table S6 and Fig. S2).

Interplay between Transposable Elements and DNA Methylation in Mycena. Similar to other fungal genomes $(22,23)$, much of the variation in the Mycena nuclear genome sizes can be explained by repetitive DNA content (SI Appendix, Table S7). Only $11.7 \%$ of the smallest genome ( $M$. chlorophos) was repeats, which is in stark contrast to the $39.0 \%$ and $35.7 \%$ in M. sanguinolenta and M. venus, respectively. The majority of transposable elements (TEs) in Mycena were long terminal repeat (LTR) retrotransposons (60 to $85 \%$ ), followed by DNA transposable elements (11 to 24\%) (Fig. $1 E$ and SI Appendix, Table S7). Interestingly, the larger genomes of $M$. sanguinolenta and $M$. venus contained the lowest proportion of LTRs (24.9 and $31.1 \%$, respectively), but the highest proportion of unclassified repeats (55.4 and 50.3\%, respectively) (SI Appendix, Table S7). A total of 16.6 to $36.5 \%$ of the unclassified repeat families shared 53.8 to $60.5 \%$ nucleotide identity with known transposable elements, suggesting they were degenerated copies which we defined as relic TEs (SI Appendix, Table S8). Fig. $2 A$ shows that the largest assembled chromosome of $M$. indigotica exhibits high proteincoding gene content and low transposable element density at scaffold centers, which is typical of fungal chromosomes $(24,25)$. Such observations were consistent across large Mycena scaffolds (typically $>1 \mathrm{Mb}$ ), suggesting that our assemblies were robust enough to capture evolutionary dynamics across chromosomes.

We detected 5-methylcytosine $(5 \mathrm{mC})$ DNA methylation levels across the five Mycena assemblies with nanopore long reads using deepsignal (26) which was initially trained with $M$. kentingensis bisulphite sequences (Methods). CG sites were found either highly (mCG level $>60 \%)$ or weakly $(<15 \%)$ methylated in gene body, displaying a bimodal distribution (SI Appendix, Fig. S4). Such a bimodal distribution has also been observed in plants, animals, and other fungi, including Tuber melanosporum and Pseudogymnoascus destructans (27-32). Within Mycena, the CG methylation in genes (5.4 to $10.5 \%$ ) was much lower than that in repeats-i.e., TEs and unclassified repeats (11.6 to $84.5 \%$ ) (Fig. $2 B$ and SI Appendix, Table S9). The level of CG methylation in these genomes is comparable with those of a previous survey on DNA methylation in 528 fungal species (32), which revealed that $5 \mathrm{mC}$ levels were highest in Basidiomycota, further indicating that DNA methylation has a specific effect on repeats in Mycena genomes. DNA transposons or LTR were enriched in $5 \mathrm{mC}$ levels and were higher than flanking regions (SI Appendix, Fig. S5). Except for DNA transposons in M. kentingensis, LTR retrotransposons had the highest CG methylation levels of all types of transposable elements (Fig. $2 B$ ). Furthermore, CG methylation in relic TEs was clearly lower than that in classic TEs (SI Appendix, Table S9). Among the Mycena species, we found that $M$. sanguinolenta and $M$. venus with larger genomes and higher repeat content had lower levels of methylation in the repeats, and the repeat methylation was much higher in $M$. indigotica, M. chlorophos, and M. kentingensis, which have smaller genomes (Fig. $2 C$ ). The same pattern was also observed in genes, though they had fewer changes in their methylation level than did repeats. Our results indicate that the variant composition of repeats is differentially mediated by DNA methylation among closely related Mycena species. Hence, genome expansion in Mycena was likely a result associated with transposable element proliferation and the accumulation of relic TEs, which yielded reduced methylation in active copies; this is also observed in some plants, e.g., Arabis alpina (33) and Manihot esculenta (34).

A Single Origin of Bioluminescent Fungi in the Ancestor of Mycena and the Marasmioid Clade. Phylogenomic analyses based on singlecopy ortholog sets have placed Mycena sister to the marasmioid clade, including Armillaria and Omphalotus, which are the other two lineages in which bioluminescent species have been identified. This species phylogeny was recovered in both maximum likelihood analysis (35) of a concatenated supermatrix of singlecopy gene alignments (Fig. $1 B$ ) and coalescent-based analysis using 360 gene trees (36) (SI Appendix, Fig. S6). In our four bioluminescent Mycena species, we identified genes involved in luciferin biosynthesis and their orthologs across species (Fig. 1C). Fig. $1 D$ shows phylogenetic reconciliation, which suggests that the orthogroup containing luciferases was present in the last common ancestor of the mycenoid + marasmioid clade and Schizophyllaceae, predating their incorporation into the luciferase cluster. This is in contrast to a previous report (7) suggesting that luciferase originated in the last common ancestor of the Agaricales. Phylogenies of other members of the luciferase cluster were also congruent with the species tree (SI Appendix, Fig. S7 $A-D$ ). Using MCMCtree (37) with three fossil calibrations, we estimated the age of mycenoid most recent common ancestor to be 105 to 147 million years ago (Mya) in the Cretaceous (Fig. $1 B$ ). This is consistent with recent estimates [78 to 110 (8) and mean 125 (1) Mya] and overlaps with the initial rise and diversification of angiosperms (38), suggesting that Mycena are ecologically associated with plants acting as saprotrophs or mycorrhizal partners (3). Finally, the age of mycenoid and marasmioid which was also the age of the luciferase cluster in fungi was estimated to originate around 160 million years ago during the late Jurassic (Fig. 1B).

Differential Conservation of Synteny Regions across Mycena Genomes. We attempted to characterize chromosome evolution in the mycenoid clade using the newly available, highly contiguous assemblies for Mycena. We first compared the patterns of 4,452 single-copy ortho$\log$ pairs between assemblies of $M$. indigotica and Armillaria ectypa (SI Appendix, Fig. S8). The majority of scaffolds between the two species could be assigned one-to-one relationships unambiguously, providing strong evidence that macrosynteny has been conserved between the marasmioid and mycenoid clades. Such chromosomelevel synteny remained conserved until the last common ancestor of the Agaricales, when $M$. indigotica was compared against the genome of Pleurotus ostreatus (SI Appendix, Fig. S9). Based on the 

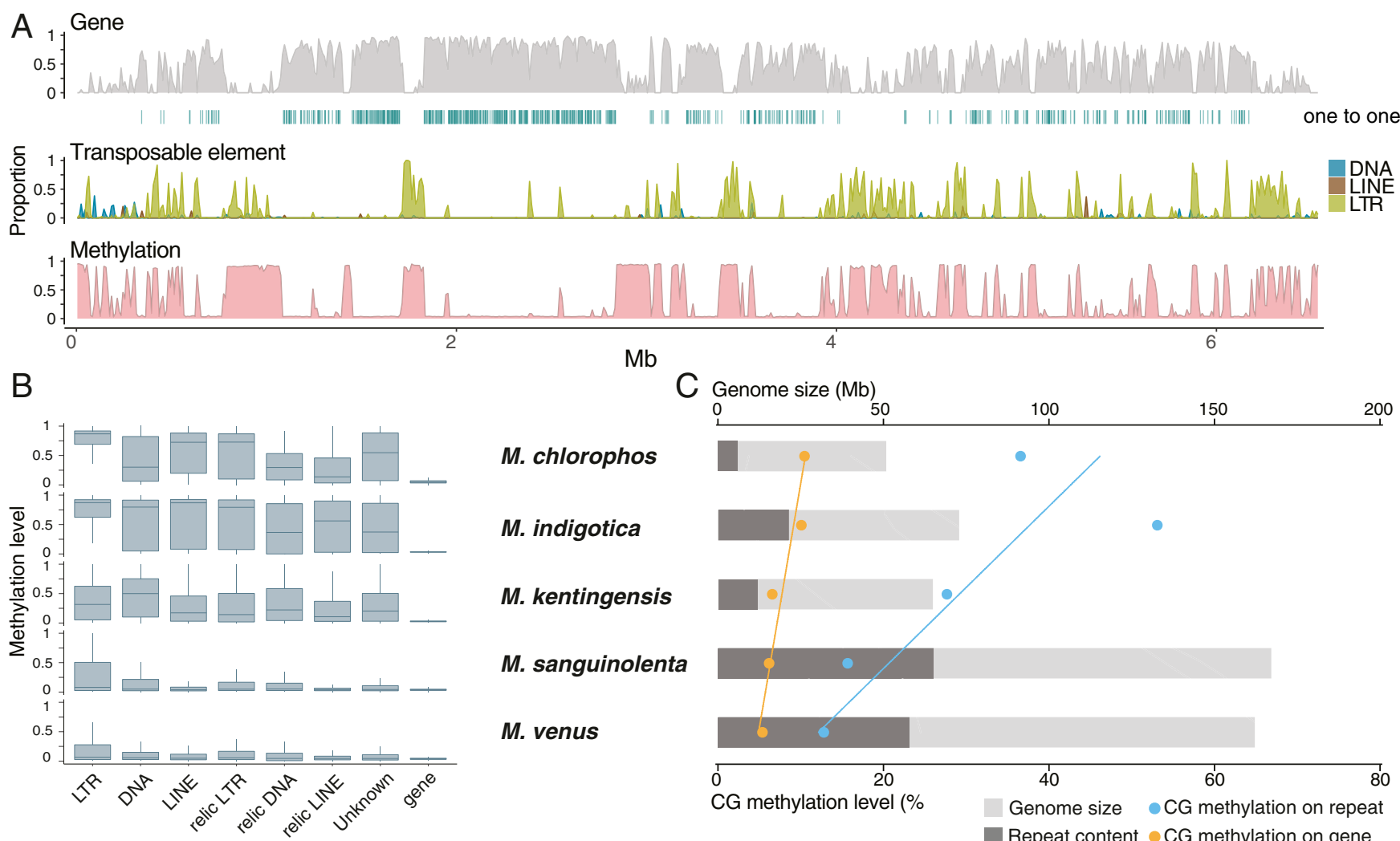

M. chlorophos

M. indigotica

M. kentingensis

M. sanguinolenta

M. venus

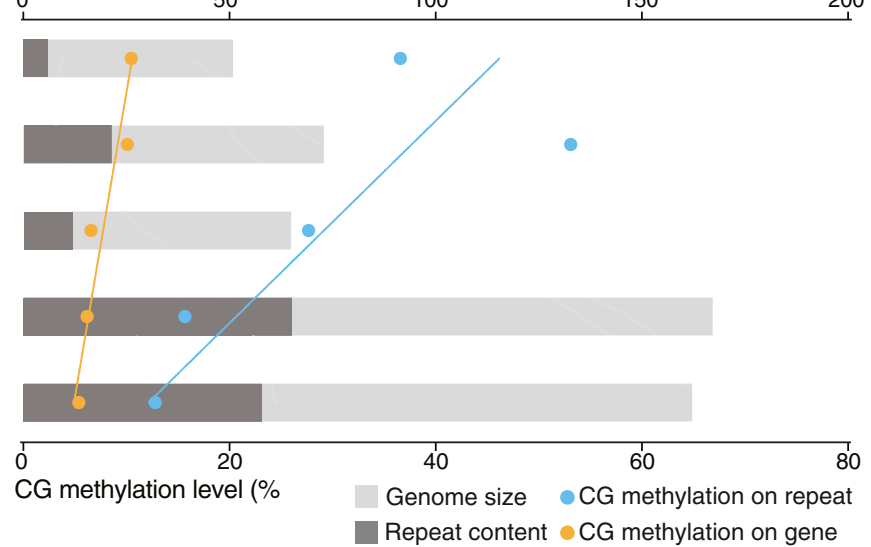

Fig. 2. Distribution of Mycena genome features. (A) M. indigotica chromosome one. For every nonoverlapping 10-kb window, the distributions from Top to Bottom are: 1) Gene density (content in percentage). Blue stripes denote positions of single-copy ortholog with $M$. chlorophos. 2) Density of TEs, including LTRs, LINES, and DNA. 3) Average methylation level called from CpG sites per window. The high methylation window generally clustered in high TE regions with low gene density. $(B)$ Methylation level in genes and different types of repeats. (C) Relationships among genome size, number of repeats, and CG methylation levels in Mycena.

clustering of single-copy orthologs, we identified 10 linkage groups resembling the number of known karyotypes in Basidiomycota, suggesting possible ancestral chromosome numbers (25).

The $M$. indigotica scaffolds exhibit high orthologous gene density in the centers of scaffolds (Fig. $2 A$ ). Fungal chromosomes can typically be compartmentalized into chromosomal cores and subtelomeres which display differential evolutionary dynamics $(24,39)$. In some extreme cases, filamentous pathogenic fungi contain entire lineage-specific chromosomes that are gene sparse and enriched in transposable elements (40). In the case of

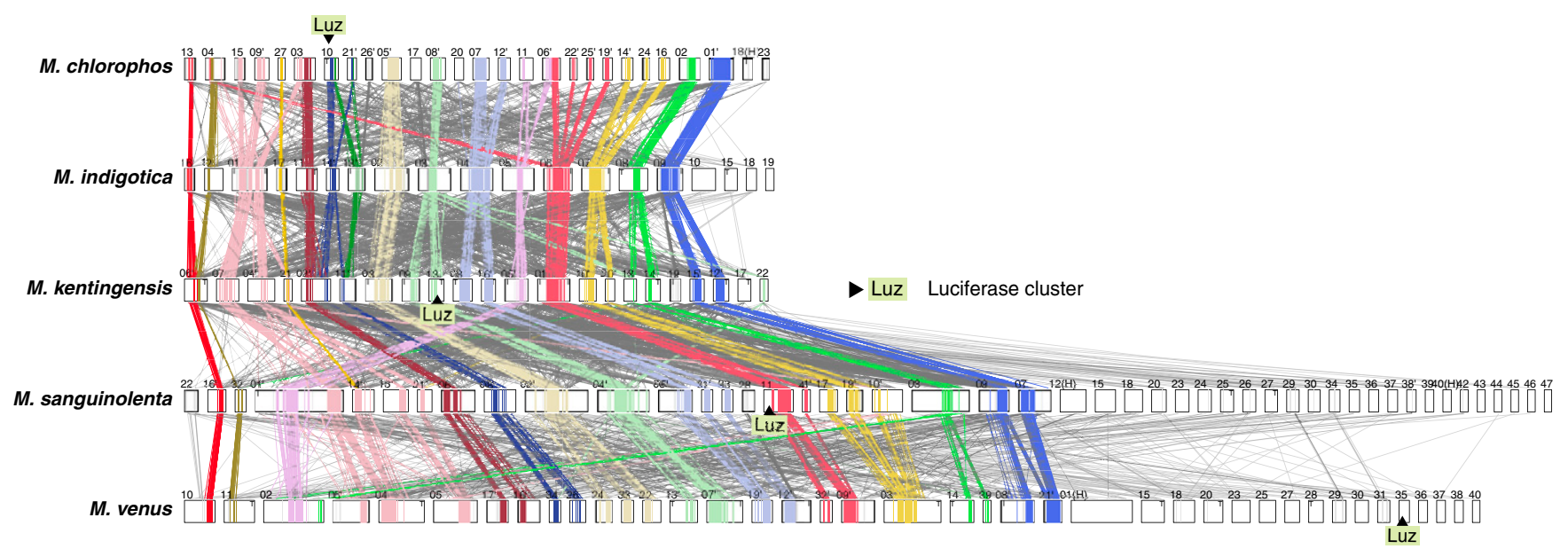

Fig. 3. Genome synteny in Mycena genomes. Schematic representation of the interscaffold relationship between species. The lines between scaffolds denote single-copy orthologs between a pair of species. Shaded areas in each scaffold denote high-synteny regions defined by DAGchainer (41) and colors denote linkage groups assigned by most abundant pairwise single-copy orthologs. Lines are color coded according to corresponding linkage groups. Black triangles denote locations of luciferase clusters. 
Mycena, a multigenome comparison showed that synteny conservation was typically either lost at the scaffold ends or extended by several megabases across the assemblies (Fig. 3).

Defining precise boundaries between regions with and without synteny is challenging. Based on the clustering of orthologous genes using DAGchainer (41), we partitioned the scaffolds into low and high synteny regions. As expected, highly syntenic regions in Mycena were typically found at the scaffold centers. In contrast, synteny was not identified in part of scaffolds or, in some cases, throughout the entire scaffolds, as was the case for the largest $(12.0 \mathrm{Mb})$ assembled scaffold of M. venus (Fig. 3). These regions are highly enriched in repeats; they have 1.5 to 2.6-fold higher methylation levels and are overrepresented in expanded and contracted OGs compared to high synteny regions (SI Appendix, Fig. S10 and Table S10; two-proportion $z$ test, $P<$ 2.1E-9). Expansions and contractions of gene families were 1.8to 4.2- and 1.4- to 2.9-fold higher in the low than high synteny regions, respectively; differential gain and loss of genes in these regions may have important implications for Mycena.

Evolutionary Dynamics of Luciferase Clusters. One of the outstanding questions surrounding the evolution of fungal bioluminescence is why bioluminescent species are scattered across the mycenoid and marasmioid clades. The mechanism of fungal bioluminescence is homologous across species (6), and this implies that nonbioluminescent mycenoid and marasmioid species must have lost the functional luciferase gene cluster. To investigate the evolutionary dynamics of the luciferase cluster, we examined all highly contiguous assemblies across the bioluminescent lineages available and inspected adjacent synteny (Fig. 4). The majority of the Mycena luciferase clusters included luciferase (luz), hispidin3-hydroxylase $(h 3 h)$, cytochrome P450 (cyp450), and hispidin synthase (hisps). We found that physical linkage was only maintained within the luciferase cluster, and synteny was lacking in genes surrounding the luciferase cluster (Fig. 4) of species in the mycenoid and Omphalotus lineages. Coupled with the aforementioned synteny analysis, we hypothesized that the luciferase cluster residing in a fast-evolving genomic region may result in it frequently being lost. The nearest TE sequence adjacent to luciferase cluster in Mycena species were 2 to $8.9 \mathrm{~kb}$ away and separated by 0 to 5 genes, suggesting possible roles of transposons mediating rearrangements (Fig. 4). Additionally, the luciferase cluster of different Mycena species was identified in low synteny regions of different linkage groups (Fig. 3), providing evidence that the location of the cluster had been extensively rearranged. In contrast, the genes surrounding the luciferase cluster among the eight Armillaria species were generally in the same order, with collinearity partially lost only in Guyanagaster necrorhiza (a very close relative of Armillaria, Fig. 4). We found that the synteny surrounding the Armillaria luciferase cluster was maintained since the common ancestor of Agaricales (SI Appendix, Fig. S11). The up- and downstream regions of the luciferase cluster belonged to two separate regions of the same ancestral chromosome linkage group, suggesting that these regions were previously rearranged-including the luciferase cluster-and were subsequently retained (SI Appendix, Fig. S11).

These observations led us to propose a most plausible evolutionary scenario in which the luciferase cluster evolved across all available bioluminescent fungi (Fig. 5). We inferred that the ancestral luciferase cluster consisted of luz, h3h, cyp450, and hisps, with caffeylpyruvate hydrolase $c p h$-involved in oxyluciferin recycling $(6,7)$ - also present on the same chromosome. This combination was found in 14 of the 15 bioluminescent species used in this study. Our data outline two contrasting scenarios by which the luciferase cluster was retained. First, the luciferase clusters in family Physalacriaceae are located in slowevolving chromosomal regions, resulting in all members of the Armillaria synteny retaining both many of the genes adjacent to

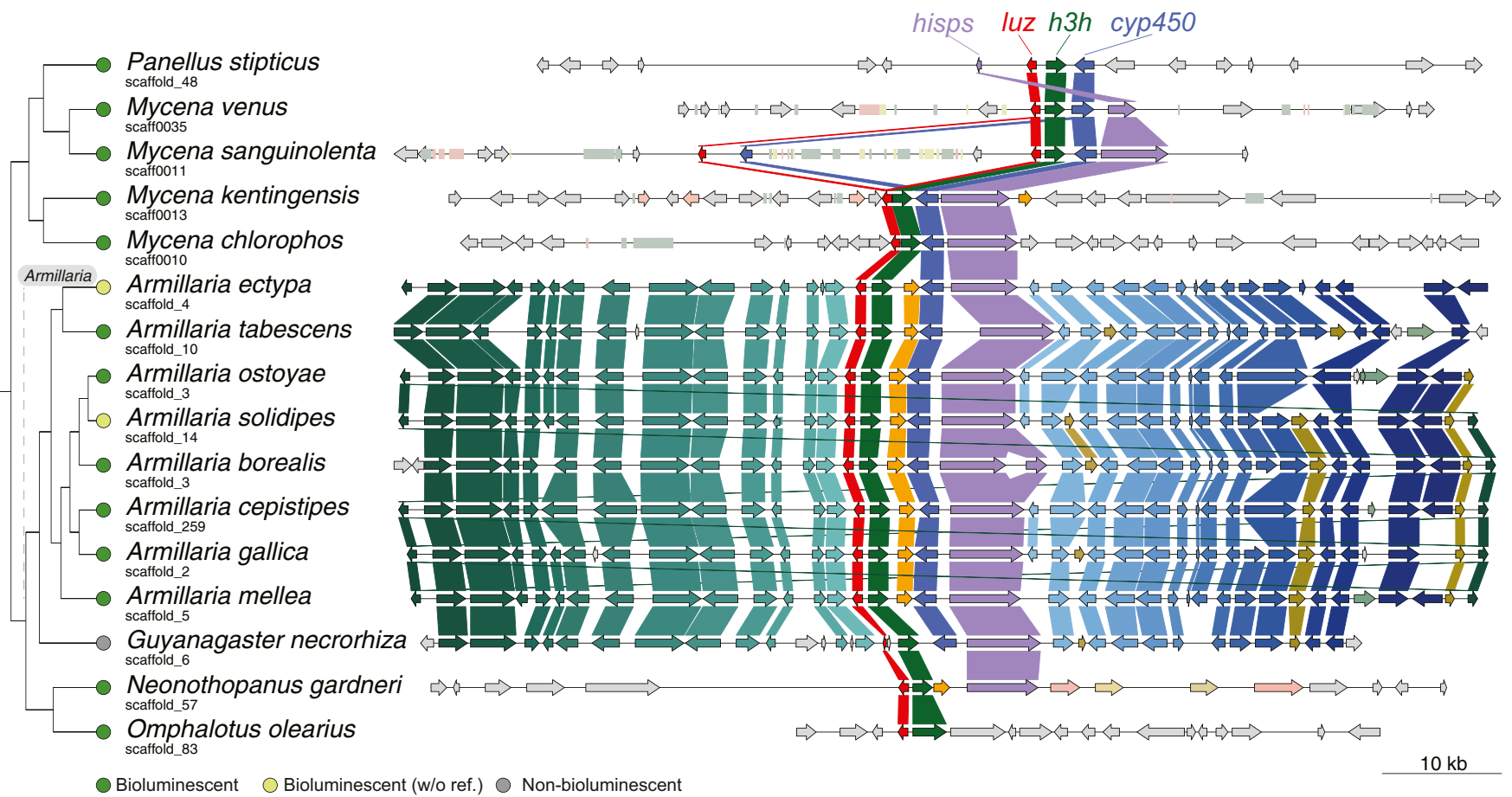

Fig. 4. Synteny around the luciferase cluster among bioluminescent fungi. The OGs shared by at least two species are labeled with the same color, regardless of their orientation. Arrows and rectangles denote protein-encoding genes and transposable element, respectively. Different colors of rectangles denote TE types (pink: LINE and LINE relic; light green: LTR and LTR relic; yellow: DNA and DNA relic). The $c p h$ gene in some species was located in other scaffolds (SI Appendix, Fig. S12). 


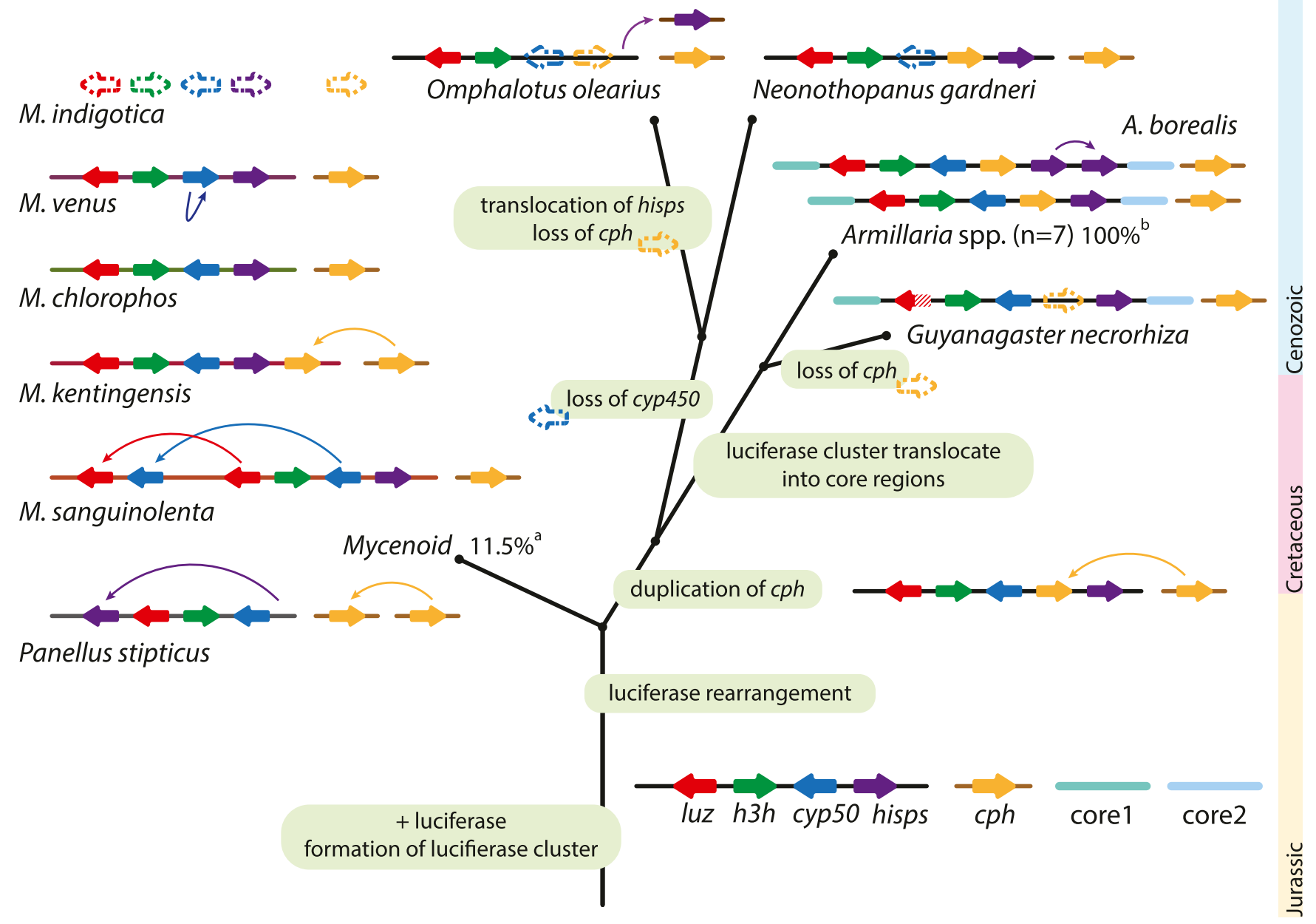

Fig. 5. Evolutionary scenario for luciferase cluster evolution. The formation of the luciferase cluster originated at the dispensable region of the last common ancestor and was susceptible to translocate to different genomic locations through rearrangement. In the ancestor of marasmioid, cph was duplicated and translocated into the luciferase cluster. Before the ancestor of the Physalacriaceae family emerged, the luciferase cluster was translocated into the core region and have since kept its synteny in the Armillaria lineage. In the most recent common ancestor of Mycena species, the luciferase cluster was located in the dispensable region and have since been susceptible to further rearrangement. Arrow box indicates gene. The dashed arrow box denotes the loss of gene. Fishhook arrow denotes translocation event. ${ }^{a}$ Percentage of bioluminescent fungi found in the mycenoid lineage (5). ${ }^{b}$ Percentage of bioluminescent fungi found in Armillaria lineage (47).

the luciferase cluster and the uniform luciferase cluster in their genomes. By residing in slow-evolving regions, the luciferase cluster in Armillaria might not be prone to losses by frequent chromosomal events (rearrangements, TE activity, etc.), explaining why it is conserved in the genus. On the other hand, the luciferase cluster in the mycenoid lineage is located in a highly dynamic genomic partition with low synteny (Fig. 3), which could explain why Mycena fungi had a higher tendency to lose the luciferase cluster compared to Armillaria species.

Variations were common in the luciferase cluster. $c p h$ was located in different scaffolds in four of the five Mycena species (SI Appendix, Fig. S12). In M. sanguinolenta, luz and cyp450 were duplicated adjacent to the luciferase cluster (Fig. 4). Losses were observed at different positions in the phylogeny. The nonbioluminescent $M$. indigotica lost the entire luciferase cluster, but $h 3 h$ homologs were found in other regions of the genome, while $G$. necrorhiza has a partial luciferase (7) and three other enzymes (Fig. 4), suggesting that an independent loss of luciferase function alone was enough for it to lose its bioluminescence. Interestingly, we found that the $c p h$ gene was independently translocated adjacent to the luciferase cluster in both $M$. kentingensis and the ancestor of the marasmioid clade (SI Appendix, Fig. S13); it was presumably favored and maintained here by natural selection (42) A selection analysis of genes in the luciferase cluster revealed that 23.1 to $45.2 \%$ of conserved sites exhibit purifying selection, with only 7 to 28 sites under episodic selection (SI Appendix, Fig. S14).

Expression Profile of Luciferase Cluster and Identification of Conserved Genes Involved in Fungal Bioluminescence. Fungal bioluminescence is believed to have ecological roles, such as attracting insects, and is regulated by circadian rhythms (43); however, the complete repertoire of genes involved in bioluminescence is still unknown. We carried out transcriptome profiling between mycelia with different bioluminescent intensities in four Mycena species, and identified genes that were either differentially expressed or positively correlated with bioluminescent intensities (Methods). There were 29 OGs found to contain up-regulated gene members in all four Mycena species (Fig. 6A), including luz, $h 3 h$, and hisps, consistent with bioluminescence intensity dependent on the expression of these three genes in the luciferase cluster. In particular, luz expression was significantly different between two tissues with relative high and low bioluminescence in $M$. kentingensis (log fold change $[\log \mathrm{FC}] 3.0$; adjusted $P<0.001)$ and $M$. chlorophos $(\log \mathrm{FC} 4.7$; adjusted $P<0.001)$; there was also a 


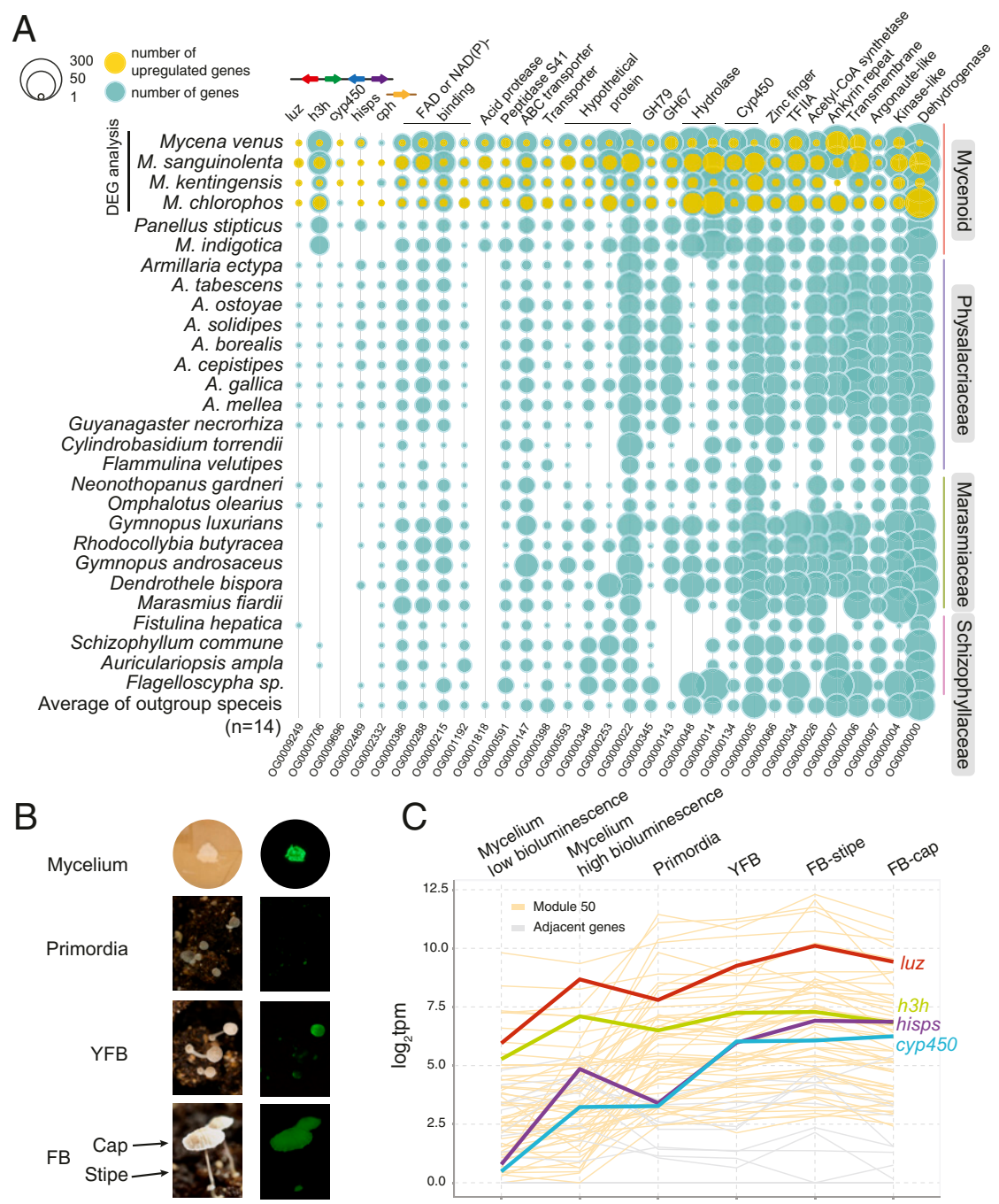

Fig. 6. Expression analysis to identify genes involved in bioluminescence. (A) Conserved up-regulated OGs. DEGs between mycelia with different bioluminescent intensities were identified in four bioluminescent Mycena species, and all 29 OGs-except OG0009249 and OG0000706-contain at least one upregulated gene. A detailed annotation of the genes in the OGs is listed in SI Appendix, Table S11. (B) Tissues used for transcriptomic data analysis in M. kentingensis. The Left and Right sides are the tissues under light and dark conditions, respectively (captured by a Nikon D7000). The camera setting for each tissue: mycelium, Sigma 17- to 50-mm ISO100 f2.8 with 16-min exposure time; primordia, AF-S Micro Nikkor 60-mm ISO800 f/11 with 122.4-s exposure time; YFB, AF-S Micro Nikkor 60-mm ISO800 f/11 with 60.6-s exposure time; FB, AF-S Micro Nikkor 60-mm ISO800, f/11 with 9.3-s exposure time. YFB, young fruiting body $(0.5$ to $1 \mathrm{~cm})$. FB, mature fruiting body $(>1 \mathrm{~cm})$. FB-cap, cap from FB. FB-stipe, stipe from FB. (C) Expression profile of luciferase cluster across developmental stages of $M$. kentingensis. Bold lines indicate four genes in the luciferase cluster. These four genes and the other 53 genes (yellow) were assigned into the same module (Module50) with similar expression patterns. The genes located up- or downstream (gray) of the luciferin biosynthesis cluster had lower expression levels than the four genes in the cluster.

significant correlation between bioluminescent intensity and expression level in $M$. sanguinolenta (Pearson's correlation coefficient $[\mathrm{PCC}] 0.82 ; P<0.005)$ and $M$. venus $(\mathrm{PCC} 0.86, P<0.005$ Dataset S3). In M. chlorophos, however, its cyp 450 and $h 3 h$ were not differentially expressed, and four distant homologs of $h 3 h$ were found to be up-regulated (SI Appendix, Fig. S7A). Although a second copy of luz and cyp 450 were found in M. sanguinolenta, they showed much lower expression ( 2 and 3 transcripts per million [TPM], respectively) than those in the cluster (282 and 138 TPM, respectively). The remaining OGs up-regulated in mycelia showing higher bioluminescence included ABC transporters and acetyl-CoA synthetases which also showed a predicted function in metabolic adaptations to bioluminescence in firefly and glowworm $(44,45)$ (Fig. $6 A$ and SI Appendix, Table S11). In particular, four OGs were annotated as FAD or NAD(P)-binding domain-containing proteins. As these genes do not bear sequence similarity to $h 3 h$ which is also a NAD $(\mathrm{P}) \mathrm{H}-$ dependent enzyme, they are likely involved in other biochemical processes that are required during bioluminescence.

Differences in bioluminescent intensity have been recorded in tissues of fungi both in nature (4, 5, 46-48) and-for $M$. kentingensis-in a laboratory environment, in which the life cycle can be completed (Fig. 6B). To investigate putative roles of bioluminescence across developmental stages, additional transcriptome profiling was carried out in the primordia, young fruiting body, and cap (pileus) and stipe of the mature fruiting body of $M$. kentingensis. Bioluminescence was stronger in the cap than in the stipe, so we expected the luciferase cluster genes to have higher expression in the cap tissue. However, luz and $h 3 h$ showed opposite expression patterns (Fig. 6C and Dataset S4), suggesting that there may be other regulators involved in bioluminescence in $M$. kentingensis. 
The regulation of bioluminescence in M. kentingensis during development was determined by performing a weighted correlation network analysis (WGCNA) $(49,50)$, which identified 67 modules of coexpressed genes in these stages (SI Appendix, Fig. $\mathrm{S} 15)$. All members of the luciferase cluster luz, $h 3 h$, cyp 450 , and hisps belonged to the same module (Module50; Fig. 6C) of 57 genes, suggesting that the expression of the luciferase cluster members are coregulated during developmental stages. Only two genes belonging to OG0001818 (acid protease) and OG0000000 (short-chain dehydrogenase) which were part of the 29 aforementioned OGs associated with bioluminescence in the mycelium samples across Mycena. Six genes in this module were annotated as carbohydrate-active enzymes (Dataset S5): one GH75 (chitosanase), one AA1_2 (laccase; ferroxidases), two GH16, and two genes with two $\bar{C} B M 52$ domains. GH16 (glucanases) and AA1 (laccases) are known to be differentially expressed during fruiting body development (51), implying a possible link between cell wall remodelling during development and bioluminescence. In addition, we reanalyzed the transcriptomes of Armillaria ostoyae across different developmental stages from Sipos et al. (9). Consistent with the observation that bioluminescence was only observed in mycelia and rhizomorphs in A. ostoyae (52, 53), the expressions of luz, h3h, cyp450, and cph were highest in these tissues (SI Appendix, Fig. S16). Together, these results imply that the luciferase cluster was differentially regulated during development and that the extent of the expressions was also different among bioluminescent species of different lineages.

Gene Families Associated with the Evolution of Mycenoid Species. We assessed orthologous group evolution by analyzing OG distribution dynamics along a time-calibrated phylogeny using CAFE (54). The rate gene family changes in mycenoid were comparable to those of other branches of Agaricales (likelihood ratio test; $P=0.25)$. A total of 703 orthologous groups were expanded at the origin of the mycenoid lineage (SI Appendix, Fig. S17). Analysis of gene ontology terms showed that these genes were enriched in NADH dehydrogenase activity, monooxygenase activity, iron ion binding, and transferase activity (Dataset S6). Additionally, we sought to identify proteins specific to mycenoid species by annotating protein family (Pfam) domains and comparing them with those of species outside this lineage (Dataset S7). A total of 537 Pfam domains were enriched in the mycenoid lineage (onefold by Wilcoxon rank sum test with $P<0.01$; Dataset S8) of which 3 to 17 were species specific. Acyl_transf_3 (acyltransferase family; PF01757), contained in a range of acyltransferase enzymes, was the only domain found in all six mycenoid species. The closest homologs were found in ascomycetous Cadophora, Pseudogymnoascus, or Phialocephala (31 to $35 \%$ identity with 73 to $100 \%$ coverage). Four of the enriched domains are known pathogenesis-related domains expanded in pathogenic Agaricales Moniliophthora (55) and Armillaria species (9): COesterase (PF00135; carboxylesterase family), thaumatin (PF00314), NPP1 (PF05630; necrosis-inducing protein), and RTA1 (PF04479; RTA1-like protein) (SI Appendix, Fig. S18). Moreover, M. sanguinolenta and $M$. venus contained over 100 and 17 copies of COesterase and thaumatin (median 37 and 4 copies in other fungal species of this study), respectively.

\section{Discussion}

Bioluminescence is one of the most unusual and fascinating traits in fungi, but the evolutionary history of the luciferase gene cluster, which underlies this phenomenon, has remained elusive. Here, we produced highly contiguous genome assemblies using nanopore technology and annotations for five Mycena species to examine their genome dynamics and evolutionary histories of fungal bioluminescence. The results of phylogenomic analyses on these genomes have important implications for the origin of luciferases.

The first question we addressed is whether fungal bioluminescence originated once or multiple times. Our species phylogeny is in good general agreement with comparative genomic analyses around this group (9). We show that the fungal luciferase, which represents a de novo origin of luciferase activity different from that in other lineages (insects, bacteria, etc.), first appeared together with other members of the luciferase cluster in the last common ancestor of mycenoid and marasmioid clades. Compared to previous inferences that this cluster had a single origin, our results imply extensive loss of the luciferase cluster in these two clades (Fig. $1 B$ and $C$ ) to explain the patchy phylogenetic distribution of and minor presence for bioluminescence in fungi. An alternative scenario could be that fungal bioluminescence arose multiple times through convergent evolution. Although multiple origins yield a more parsimonious model, we can confidently reject this hypothesis because both the fungal luciferase and the luciferase gene cluster are clearly homologous across distant bioluminescent fungi (6). Although a single origin and the excessive number of implied losses may appear counterintuitive, models of trait evolution and recent empirical evidence of phylogenetically patchy, but homologous traits $(56,57)$ has emerged offering a biologically reasonable explanation. One attractive model is the latent homology model (58), which posits that precursor traits can potentiate lineages for easily and recurrently evolving similar traits. More comprehensive surveys of genomes in these lineages are needed to make informed speculations on whether latent homologies might have facilitated the evolution of bioluminescence in fungi.

The next outstanding question therefore is what caused the frequent losses of bioluminescence in fungi? Our evolutionary reconstructions show that the luciferase cluster might have originated in a low-synteny region of genomes (Fig. 3), making it susceptible to rearrangement, which suggests it is highly prone to loss and explains why most mycenoid and marasmioid species are nonbioluminescent. This is consistent with a previous report that the main evolutionary process in fungal gene clusters is vertical evolution followed by differential loss (59). Interestingly, synteny was retained in luciferase clusters and adjacent genes of Armillaria species (Fig. 4), which are better known for their roles as plant pathogens (9). Such synteny remained detectable when compared to Mycena, representative Agaricales, and $P$. ostreatus genomes, suggesting that in Armillaria, the luciferase cluster was translocated to a region of the genome where synteny was maintained since the common ancestor of Agaricales and $P$. ostreatus. Indeed, bioluminescence was identified in all nine examined Armillaria species (47). One alternative but less parsimonious scenario would be that the luciferase cluster originated in a high-synteny region and subsequently translocated to lowsynteny regions in ancestors of both mycenoid and various families within the marasmioid clade. The repeated duplication and relocation of $c p h$ that we observed in the luciferase cluster is under selection pressure, suggesting that bioluminescence was maintained in fungi that still exhibit this phenotype. A systematic quantification of bioluminescence and more complete genome assemblies will help reconstruct the evolutionary events that contributed to the polymorphism and functional diversity in the luciferase clusters.

Researchers have long been puzzled over the ecological role of bioluminescence in fungi. One explanation that has been put forth for Neonothopanus gardneri is that bioluminescence follows a circadian rhythm to increase spore dispersal by attracting arthropods in the evening (43). If true, this is most likely a derived adaptation, as most bioluminescent fungi-including Mycena, Omphalotus, and Armillaria species-disperse spores via wind, display bioluminescence continuously, and are not known to attract insects (60). Besides, attraction is insufficient to explain 
luminescence in the mycelium. We have shown that the luciferase cluster in M. kentingensis is constitutively expressed throughout development. We further identified a handful of genes whose expressions are correlated with fungal bioluminescence and may therefore be candidates for experimental follow-up studies (Fig. 6). If fungal bioluminescence originated as a byproduct of a biological process that is currently unknown, the ecological role was likely to be initially limited, which may explain why it has undergone subsequent losses in many species. For those that have retained bioluminescence, its ecological role remains unknown, but we speculate that it may be species specific, explaining why the luciferase cluster had been maintained across hundreds of millions of years.

In summary, our comparative analyses allowed us to propose an evolutionary model pinpointing changes in the luciferase cluster across all bioluminescent fungi with published genomes. Our findings offer insights into the evolution of a gene cluster spanning over 160 million years and suggest that the retained luciferases were under strong purifying selection. Our Mycena genome sequences may complement ongoing research on the application of bioluminescent pathways (7) and shed light on the ecological role of bioluminescence in fungi.

\section{Methods}

More detailed information on the materials and methods used in this study are provided in SI Appendix.

De Novo Assemblies of Mycena Species. Haploid genome length and heterozygosity of the five Mycena species (M. kentingensis, M. venus (61), M. san guinolenta, $M$. indigotica, and $M$. chlorophos) were estimated from Illumina reads using GenomeScope (14) (ver. 2.0). Oxford nanopore reads were as sembled using the Canu (13) (ver. 1.8) assembler. Consensus sequences of the assemblies were polished first by five iterations of Racon (62) (ver. 1.3.2) followed by Medaka (ver. 0.7.1; https://github.com/nanoporetech/medaka) using Oxford nanopore reads. HaploMerger2 (63) (ver. 20180603) was then run on to generate haploid assemblies. Finally, the consensus sequences were further corrected with Illumina reads using Pilon (64) (ver. 1.22). QVs of the final as semblies were calculated as described in Koren et al. (65). Throughout each stage the genome completeness was assessed using fungi and the basidiomycete dataset of BUSCO (19) (ver. 4.1.2). Putative telomeric repeats were searched for copy number repeats of fewer than 10 mers using Tandem Repeat Finder (66) (ver. 4.09; options: 277801050 500). The hexamer TTAGGG was identified (SI Appendix, Table S12).

Gene Predictions and Functional Annotation. Protein sequences from Uniprot fungi $(32,991$ sequences; downloaded December 20, 2018) and Coprinopsis cinerea, P. ostreatus PC15 (v2.0), Schizophyllum commune, and Armillaria mellea from MycoCosm (67) portal were downloaded as reference proteomes. Transcriptome reads were first mapped to the corresponding genome assemblies using $\operatorname{STAR}(68,69)$ (ver. 2.5.3a) and subsequently assembled into transcripts using Trinity (70) (ver. 2.3.2; guided approach) Stringtie (71) (ver. 1.3.1c), CLASS2 (72) (ver. 2.1.7), and Cufflinks (73) (ver 2.2.1). The samples used for input are listed in Dataset S1. Transcripts generated from Trinity were aligned to the references using GMAP (74). All transcripts were merged, filtered, and picked using MIKADO (75) (ver. 1.1) The gene predictor Augustus (76) (ver. 3.2.1) and gmhmm (77) (ver. 3.56) were trained using BRAKER2 (78) (option fungi and softmasked), and SNAP (79) was trained using the assembled transcripts with MAKER2 (18) (ver 2.31.9). The assembled transcripts, reference proteomes, and BRAKER2 annotations were combined as evidence hints for input in the MAKER2 (18) annotation pipeline. MAKER2 (18) invoked the three trained gene predictors to generate a final set of gene annotation. Descriptions of amino acid sequences of the proteome were annotated using Blast2GO (80) and gene ontology (GO) terms were annotated using Argot (ver.2.5 (81). A total of 80.0 to $86.7 \%$ of proteomes were assigned at least one GO term. Genes encoding carbohydrate-active enzymes were identified using dbCAN (82) (database ver. Hmm9.0; code ver. 2.0.0) by searching for sequence homologs with HMMER (83). Consensus (library) sequences of repetitive elements were identified using the pipeline described in Berriman et al. (84).

Methylation Analyses. High-quality paired-end reads were aligned to the genome assemblies of $M$. kentingensis using the bisulfite-specific aligner BS-
Seeker2 (85). Only uniquely mapped reads were retained. The cytosines covered by at least four reads were included in the data analysis, and the DNA methylation level for each cytosine was estimated as \#C/(\#C+\#T), where \#C is the number of methylated reads and \#T is the number of unmethylated reads.

One or two nanopore flowcells for each Mycena species were selected to infer methylation information using deepsignal (26) (ver. 0.1.5) (M. kentingensis: FAH31207, M. chlorophos: FAH31470, M. indigotica: FAH31228, $M$. sanguinolenta: FAK22405 and FAH31211, and M. venus: FAK22389 and FAH31302). The machine learning-based model was trained with one bisulfite dataset (YJMC0389) and one nanopore dataset (FAH31207) of $M$. kentingensis. The bisulfite result was first filtered for depth $>20$, then methylation levels $>0.9$ and $<0.01$ were selected for positive and negative validation datasets, respectively. All seven flowcells were called for methylation information with a customized model and default arguments. A minimal depth of 4 was applied to the results for further analysis. In the estimates of DNA methylation levels between nanopore long reads and the Illumina BS-seqs, the Pearson correlation coefficient was as high as 0.96 in the methylomes of M. kentingensis (SI Appendix, Fig. S19).

Phylogenomic Analyses. OGs among 42 species were identified using OrthoFinder $(20,21)$ (ver. 2.2.7). A total of 42 sets of amino acid sequences from 360 single-copy OGs were aligned independently using MAFFT (86) (ver. 7.271; option -maxiterate 1000). A total of three approaches were used to infer the species tree. The first two approaches relied on maximum likelihood phylogenies from individual OG alignments computed using RAxMLng (87) (ver. 0.9.0; options: -all -model LG+I+F+G4 -seed 1234 -tree pars 10 -bs-trees 100 ) with 100 bootstrap replicates. The best phylogeny and bootstrap replicates were separately used to infer a consensus tree using ASTRAL-III (36). Finally, a maximum likelihood phylogeny from the concatenated amino acid alignments of the single-copy orthogroups was constructed with 100 bootstrap replicates using RAxML-ng (87) (ver. 0.9.0; options: -all -seed 1234 -tree pars 10 -bs-trees 100 with -model LG+l+F+G4 partitioned with each OG alignment).

Estimation of Divergence Time. The divergence time of each tree node was inferred using MCMCtree in PAML (37) package [ver. $4.9 \mathrm{~g}$ with approximate likelihood (88); the JC69 model and the rest were default]. The individual amino acid alignments of 360 single-copy orthologs were converted into corresponding codon alignments using PAL2NAL (89) (ver. 14). The species tree and concatenated alignments of these single-copy orthologs were used as the input for MCMCtree. The phylogeny was calibrated using fossil records by placing soft minimum bounds at the ancestral node of: 1) marasmioid [using Archaeomarasmius legettii 94 to $90 \mathrm{Ma}$ (90); 90 was used], 2) Agaricales [using Paleoagaricites antiquus 110 to $100 \mathrm{Ma}$ (91); 100 was used], 3) Taxon A [ 99 Ma (92); 95 was used], and 4) a soft bound of $200 \mathrm{Ma}$ for the phylogeny. The entire analysis was run five times to check for convergence.

Synteny Analyses. Linkage groups (LGs) between M. indigotica and A. ectypa and between $M$. indigotica and $P$. ostreatus were assigned, based on the reciprocal majority of the single-copy orthologs ( $S I$ Appendix, Figs. S8 and S9). Scaffolds that contained fewer than 10 single-copy orthologs, shorter than $500 \mathrm{~kb}$, or shorter than species N90 were excluded from the analysis. Linkage groups within Mycena were assigned based on majority and at least $10 \%$ of single-copy ortholog links with $M$. indigotica scaffolds. Subsequent scaffolds were identified as the same linkage group if they contained a majority of pairwise one-to-one single-copy orthologs belonging to the $M$. indigotica LG. Synteny blocks of each species were estimated from pairwise proteome comparisons against its closest relative using DAGchainer (41) (options -Z 12 -D 10 -g 1 -A 5). Synteny around luciferase cluster was plotted using the genoPlotR (93) package.

RNA-Seq Analysis of Differential Bioluminescent Mycelium. Quality trimming of the RNA sequencing reads was conducted using Trimmomatic (94). The sequencing reads were mapped to the genome using $\operatorname{STAR}(68,69)$ (ver. STAR 2.5.1b modified; default parameters). Raw read counts of the gene models were quantified by FeatureCounts (95) (ver. v1.5.0; -p -s 2 -t exon). For $M$. kentingensis and $M$. chlorophos, the differentially expressed genes (DEGs) were analyzed using DESeq2 (96). Genes with logFC $>0$ and false discovery rate (FDR) $\leq 0.05$ were defined as DEGs. For $M$. sanguinolenta and $M$. venus, the DEGs were identified by the Pearson correlation coefficient between the bioluminescence intensity (relative light unit [RLU]) normalized by weight (RLU/mg) and log transformation of counts per million. Genes with correlation coefficient $>0.7$ and $P<0.01$ were defined as DEGs. 
RNA Analysis of $\boldsymbol{M}$. kentingensis and $\boldsymbol{A}$. ostoyae Developmental Stages. The reads from transcriptomes of the primordia, young fruiting body, and cap and stipe of mature fruiting body were conducted by the same method of mapping the mycelia transcriptome reads. To identify coexpressed genes among transcriptomes, the transformation of TPM from six different tissues-mycelia with high bioluminescence and low bioluminescence, primordia, young fruiting body, and fruiting body cap and stipe-were calculated. The lowest $25 \%$ expressed gene across all samples were excluded and coexpression was analyzed using WGCNA $(49,50)$ package in R (maxBlockSize $=10,000$, power $=20$, networkType $=$ signed, TOMType $=$ signed, minModuleSize $=30$ ). The lllumina reads among 10 stages from $A$. ostoyae were also downloaded from National Center for Biotechnology Information (NCBI)'s GEO Archive (accession no. GSE100213) and also analyzed by the same pipeline of $M$. kentingensis to identify coexpressed genes among the transcriptomes.

Data Availability. Raw sequences, genome assembly, and annotation of five Mycena species were deposited in the NCBI BioProject (accession no. PRJNA623720) and Genome ( $M$. chlorophos JACAZE000000000, M. indigotica JACAZF000000000, M. kentingensis JACAZG000000000, M. sanguinolenta JACAZH000000000, and M. venus JACAZI000000000) databases.

1. M. He et al., Notes, outline and divergence times of Basidiomycota. Fungal Divers. 99, 105-367 (2019)

2. C. Wei, R. Kirschner, A new Mycena species with blue basidiomata and porioid hy menophore from Taiwan. Mycoscience 60, 10-13 (2019).

3. E. Thoen et al., In vitro evidence of root colonization suggests ecological versatility in the genus Mycena. New Phytol. 227, 601-612 (2020).

4. A. Chew, D. Desjardin, Y. Tan, M. Musa, V. Sabaratnam, Bioluminescent fungi from Peninsular Malaysia-a taxonomic and phylogenetic overview. Fungal Divers. 70, 149-187 (2015)

5. A. Cortés-Pérez et al., New species and records of bioluminescent Mycena from Mexico. Mycologia 111, 319-338 (2019).

6. Z. M. Kaskova et al., Mechanism and color modulation of fungal bioluminescence. Sci. Adv. 3, e1602847 (2017)

7. A. A. Kotlobay et al., Genetically encodable bioluminescent system from fungi. Proc Natl. Acad. Sci. U.S.A. 115, 12728-12732 (2018).

8. T. Varga et al., Megaphylogeny resolves global patterns of mushroom evolution. Nat Ecol. Evol. 3, 668-678 (2019).

9. G. Sipos et al., Genome expansion and lineage-specific genetic innovations in the forest pathogenic fungi Armillaria. Nat. Ecol. Evol. 1, 1931-1941 (2017)

10. D. Floudas et al., Evolution of novel wood decay mechanisms in Agaricales revealed by the genome sequences of Fistulina hepatica and Cylindrobasidium torrendii. Fungal Genet. Biol. 76, 78-92 (2015)

11. Y. J. Park et al., Whole genome and global gene expression analyses of the mode mushroom Flammulina velutipes reveal a high capacity for lignocellulose degradation. PLoS One 9, e93560 (2014).

12. D. Liu, M. Hunt, I. J. Tsai, Inferring synteny between genome assemblies: A systematic evaluation. BMC Bioinf. 19, 26 (2018)

13. S. Koren et al., Canu: Scalable and accurate long-read assembly via adaptive $k$-mer weighting and repeat separation. Genome Res. 27, 722-736 (2017)

14. T. R. Ranallo-Benavidez, K. S. Jaron, M. C. Schatz, GenomeScope 2.0 and Smudgeplot for reference-free profiling of polyploid genomes. Nat. Commun. 11, 1432 (2020).

15. K. Shafin et al., Nanopore sequencing and the Shasta toolkit enable efficient de novo assembly of eleven human genomes. Nat. Biotechnol. 38, 1044-1053 (2020).

16. R. Heinzelmann, D. Rigling, G. Sipos, M. Münsterkötter, D. Croll, Chromosomal assembly and analyses of genome-wide recombination rates in the forest pathogenic fungus Armillaria ostoyae. Heredity 124, 699-713 (2020).

17. G. Pérez, J. Pangilinan, A. G. Pisabarro, L. Ramírez, Telomere organization in the ligninolytic basidiomycete Pleurotus ostreatus. Appl. Environ. Microbiol. 75 1427-1436 (2009).

18. C. Holt, M. Yandell, MAKER2: An annotation pipeline and genome-database management tool for second-generation genome projects. BMC Bioinf. 12, 491 (2011).

19. F. A. Simão, R. M. Waterhouse, P. loannidis, E. V. Kriventseva, E. M. Zdobnov, BUSCO: Assessing genome assembly and annotation completeness with single-copy orthologs. Bioinformatics 31, 3210-3212 (2015).

20. D. M. Emms, S. Kelly, OrthoFinder: Phylogenetic orthology inference for comparative genomics. Genome Biol. 20, 238 (2019).

21. D. M. Emms, S. Kelly, OrthoFinder: Solving fundamental biases in whole genome comparisons dramatically improves orthogroup inference accuracy. Genome Biol. 16 157 (2015).

22. S. Duplessis et al., Obligate biotrophy features unraveled by the genomic analysis of rust fungi. Proc. Natl. Acad. Sci. U.S.A. 108, 9166-9171 (2011).

23. A. Zheng et al., The evolution and pathogenic mechanisms of the rice sheath blight pathogen. Nat. Commun. 4, 1424 (2013).

24. J. E. Stajich et al., Insights into evolution of multicellular fungi from the assembled chromosomes of the mushroom Coprinopsis cinerea (Coprinus cinereus). Proc. Natl. Acad. Sci. U.S.A. 107, 11889-11894 (2010).

25. C. L. Chung et al., Comparative and population genomic landscape of Phellinus noxius: A hypervariable fungus causing root rot in trees. Mol. Ecol. 26, 6301-6316 (2017)
ACKNOWLEDGMENTS. We thank Chia-Ning Shen for lending us the luminometer for the duration of the project. We thank Bi-Chang Chen for providing the charge-coupled device camera to qualify bioluminescence. We thank Chi-Yu Chen and Jie-Hao Ou for their useful advice on culturing Mycena fungi. We are grateful to the National Center for High-Performance Computing for computer time and facilities. We are grateful to the "1000 Fungal Genomes-Deep Sequencing of Ecologically Relevant Dikarya" consortium for access to genome data. We thank Gregory Bonito, Hui-Ling Liao, Alejandro Rojas, and Rytas Vilgalys for permission to use the Flagelloscypha sp. FlaPMI526_1 assembly from the US Department of Energy Joint Genome Institute (JGI). We thank Mary Catherine Aime for permission to use the $G$ necrorhiza MCA 3950 assembly from JGI. The genome sequence data were produced by the JGI in collaboration with the user community. We thank Chia-Lin Chung, Ben-Yang Liao, and John Wang for commenting on an earlier version of the manuscript. We thank the National Center for Highperformance Computing ( $\mathrm{NCHC}$ ) for providing computational and storage resources. P.-Y.C. was supported by the Ministry of Science and Technology Taiwan, under grants 106-2311-B-001 -035 -MY3 and 108-2313-B-001-013 -MY3. H.-M.K. was supported by a Academia Sinica postdoctoral fellowship. I.J.T. was supported by Academia Sinica Career Development Award ASCDA-107-L01 and Ministry of Science and Technology, Taiwan under Grant 109-2628-B-001-023-. L.G.N. was supported by the "Momentum" Program of the Hungarian Academy of Sciences (Grant LP2019-13/2019).

26. P. Ni et al., DeepSignal: Detecting DNA methylation state from nanopore sequencing reads using deep-learning. Bioinformatics 35, 4586-4595 (2019).

27. X. Zhang et al., Genome-wide high-resolution mapping and functional analysis of DNA methylation in arabidopsis. Cell 126, 1189-1201 (2006).

28. Y. Wang, X. Wang, T. H. Lee, S. Mansoor, A. H. Paterson, Gene body methylation shows distinct patterns associated with different gene origins and duplication modes and has a heterogeneous relationship with gene expression in Oryza sativa (rice). New Phytol. 198, 274-283 (2013).

29. B. Montanini et al., Non-exhaustive DNA methylation-mediated transposon silencing in the black truffle genome, a complex fungal genome with massive repeat element content. Genome Biol. 15, 411 (2014).

30. P. A. Jones, Functions of DNA methylation: Islands, start sites, gene bodies and beyond. Nat. Rev. Genet. 13, 484-492 (2012).

31. N. Elango, B. G. Hunt, M. A. D. Goodisman, S. V. Yi, DNA methylation is widespread and associated with differential gene expression in castes of the honeybee, Apis mellifera. Proc. Natl. Acad. Sci. U.S.A. 106, 11206-11211 (2009).

32. A. J. Bewick et al., Diversity of cytosine methylation across the fungal tree of life. Nat. Ecol. Evol. 3, 479-490 (2019)

33. E. M. Willing et al., Genome expansion of Arabis alpina linked with retrotransposition and reduced symmetric DNA methylation. Nat. Plants 1, 14023 (2015).

34. H. Wang et al., CG gene body DNA methylation changes and evolution of duplicated genes in cassava. Proc. Natl. Acad. Sci. U.S.A. 112, 13729-13734 (2015).

35. A. Stamatakis, RAxML-VI-HPC: Maximum likelihood-based phylogenetic analyses with thousands of taxa and mixed models. Bioinformatics 22, 2688-2690 (2006).

36. S. Mirarab, T. Warnow, ASTRAL-II: Coalescent-based species tree estimation with many hundreds of taxa and thousands of genes. Bioinformatics 31, i44-i52 (2015)

37. Z. Yang, PAML 4: Phylogenetic analysis by maximum likelihood. Mol. Biol. Evol. 24 1586-1591 (2007)

38. R. Lupia, S. Lidgard, P. Crane, Comparing palynological abundance and diversity: Implications for biotic replacement during the Cretaceous angiosperm radiation. Paleobiology 25, 305-340 (1999)

39. J.-X. Yue et al., Contrasting evolutionary genome dynamics between domesticated and wild yeasts. Nat. Genet. 49, 913-924 (2017).

40. S. Dong, S. Raffaele, S. Kamoun, The two-speed genomes of filamentous pathogens: Waltz with plants. Curr. Opin. Genet. Dev. 35, 57-65 (2015)

41. B. J. Haas, A. L. Delcher, J. R. Wortman, S. L. Salzberg, DAGchainer: A tool for mining segmental genome duplications and synteny. Bioinformatics 20, 3643-3646 (2004).

42. A. Rokas, J. H. Wisecaver, A. L. Lind, The birth, evolution and death of metabolic gene clusters in fungi. Nat. Rev. Microbiol. 16, 731-744 (2018).

43. A. G. Oliveira et al., Circadian control sheds light on fungal bioluminescence. Curr. Biol. 25, 964-968 (2015)

44. M. L. Sharpe, P. K. Dearden, G. Gimenez, K. L. Krause, Comparative RNA seq analysis of the New Zealand glowworm Arachnocampa luminosa reveals bioluminescencerelated genes. BMC Genom. 16, 825 (2015).

45. T. R. Fallon et al., Firefly genomes illuminate parallel origins of bioluminescence in beetles. eLife 7, e36495 (2018)

46. D. E. Desjardin, A. G. Oliveira, C. V. Stevani, Fungi bioluminescence revisited. Photochem. Photobiol. Sci. 7, 170-182 (2008).

47. J. D. Mihail, Bioluminescence patterns among North American Armillaria species. Fungal Biol. 119, 528-537 (2015)

48. D. Bermudes, R. H. Petersen, K. H. Nealson, Low-level bioluminescence detected in Mycena-Haematopus Basidiocarps. Mycologia 84, 799-802 (1992).

49. B. Zhang, S. Horvath, A general framework for weighted gene co-expression network analysis. Stat. Appl. Genet. Mol. Biol. 4, (2005).

50. P. Langfelder, S. Horvath, WGCNA: An R package for weighted correlation network analysis. BMC Bioinf. 9, 559 (2008)

51. K. Krizsán et al., Transcriptomic atlas of mushroom development reveals conserved genes behind complex multicellularity in fungi. Proc. Natl. Acad. Sci. U.S.A. 116 7409-7418 (2019). 
52. E. N. Harvey, Bioluminescence (Academic Press, New York, 1952).

53. E. C. Wassink, "Luminescence in fungi." in Bioluminescence in Action, P. J. Herring, Ed. (Academic Press, London, 1978), pp. 171-197.

54. T. De Bie, N. Cristianini, J. P. Demuth, M. W. Hahn, CAFE: A computational tool for the study of gene family evolution. Bioinformatics 22, 1269-1271 (2006).

55. J. M. Mondego et al., A genome survey of Moniliophthora perniciosa gives new insights into Witches' Broom Disease of cacao. BMC Genom. 9, 548 (2008).

56. C. Guijarro-Clarke, P. W. H. Holland, J. Paps, Widespread patterns of gene loss in the evolution of the animal kingdom. Nat. Ecol. Evol. 4, 519-523 (2020).

57. A. M. C. Bowles, U. Bechtold, J. Paps, The origin of land plants is rooted in two bursts of genomic novelty. Curr. Biol. 30, 530-536.e2 (2020).

58. L. G. Nagy et al., Latent homology and convergent regulatory evolution underlies the repeated emergence of yeasts. Nat. Commun. 5, 4471 (2014).

59. M. Marcet-Houben, T. Gabaldón, Evolutionary and functional patterns of shared gene neighbourhood in fungi. Nat. Microbiol. 4, 2383-2392 (2019).

60. P. Weinstein, S. Delean, T. Wood, A. D. Austin, Bioluminescence in the ghost fungus Omphalotus nidiformis does not attract potential spore dispersing insects. IMA Fungus 7, 229-234 (2016).

61. C. C. Chang, C. Y. Chen, W. W. Lin, H. W. Kao, Mycena jingyinga, Mycena luguensis, and Mycena venus: Three new species of bioluminescent fungi from Taiwan. Taiwania 65, 396-406 (2020)

62. R. Vaser, I. Sović, N. Nagarajan, M. Šikić, Fast and accurate de novo genome assembly from long uncorrected reads. Genome Res. 27, 737-746 (2017).

63. S. Huang, M. Kang, A. Xu, HaploMerger2: Rebuilding both haploid sub-assemblies from high-heterozygosity diploid genome assembly. Bioinformatics 33, 2577-2579 (2017).

64. B. J. Walker et al., Pilon: An integrated tool for comprehensive microbial variant detection and genome assembly improvement. PLoS One 9, e112963 (2014).

65. S. Koren et al., De novo assembly of haplotype-resolved genomes with trio binning Nat. Biotechnol. 36, 1174-1182 (2018).

66. G. Benson, Tandem repeats finder: A program to analyze DNA sequences. Nucleic Acids Res. 27, 573-580 (1999).

67. I. V. Grigoriev et al., MycoCosm portal: Gearing up for 1000 fungal genomes. Nucleic Acids Res. 42, D699-D704 (2014)

68. A. Dobin et al., STAR: Ultrafast universal RNA-seq aligner. Bioinformatics 29, 15-21 (2013).

69. A. Dobin, T. R. Gingeras, Mapping RNA-seq reads with STAR. Curr. Protoc. Bioinf. $\mathbf{5 1}$ 1114 11-11 1419 (2015).

70. B. J. Haas et al., De novo transcript sequence reconstruction from RNA-seq using the Trinity platform for reference generation and analysis. Nat. Protoc. 8, 1494-1512 (2013).

71. M. Pertea et al., StringTie enables improved reconstruction of a transcriptome from RNA-seq reads. Nat. Biotechnol. 33, 290-295 (2015).

72. L. Song, S. Sabunciyan, L. Florea, CLASS2: Accurate and efficient splice variant annotation from RNA-seq reads. Nucleic Acids Res. 44, e98 (2016)

73. C. Trapnell et al., Differential gene and transcript expression analysis of RNA-seq experiments with TopHat and Cufflinks. Nat. Protoc. 7, 562-578 (2012).

74. T. D. Wu, C. K. Watanabe, GMAP: A genomic mapping and alignment program for mRNA and EST sequences. Bioinformatics 21, 1859-1875 (2005).
75. L. Venturini, S. Caim, G. G. Kaithakottil, D. L. Mapleson, D. Swarbreck, Leveraging multiple transcriptome assembly methods for improved gene structure annotation. Gigascience 7, giy093 (2018).

76. M. Stanke, A. Tzvetkova, B. Morgenstern, AUGUSTUS at EGASP: Using EST, protein and genomic alignments for improved gene prediction in the human genome. Genome Biol. 7 (suppl. 1), S11.1-S11.8 (2006).

77. V. Ter-Hovhannisyan, A. Lomsadze, Y. O. Chernoff, M. Borodovsky, Gene prediction in novel fungal genomes using an ab initio algorithm with unsupervised training. Genome Res. 18, 1979-1990 (2008).

78. K. J. Hoff, S. Lange, A. Lomsadze, M. Borodovsky, M. Stanke, BRAKER1: Unsupervised RNA-seq-based genome annotation with GeneMark-ET and AUGUSTUS. Bioinformatics 32, 767-769 (2016).

79. I. Korf, Gene finding in novel genomes. BMC Bioinf. 5, 59 (2004).

80. A. Conesa et al., Blast2GO: A universal tool for annotation, visualization and analysis in functional genomics research. Bioinformatics 21, 3674-3676 (2005).

81. M. Falda et al., Argot2: A large scale function prediction tool relying on semantic similarity of weighted gene ontology terms. BMC Bioinf. 13 (suppl. 4), S14 (2012).

82. Y. Yin et al., dbCAN: A web resource for automated carbohydrate-active enzyme annotation. Nucleic Acids Res. 40, W445-W451 (2012).

83. R. D. Finn, J. Clements, S. R. Eddy, HMMER web server: Interactive sequence similarity searching. Nucleic Acids Res. 39, W29-W37 (2011).

84. M. Berriman, A. Coghlan, I. J. Tsai, Creation of a comprehensive repeat library for a newly sequenced parasitic worm genome. Protoc. Exch., 10.1038/protex.2018.054 (2018).

85. W. Guo et al., BS-Seeker2: A versatile aligning pipeline for bisulfite sequencing data. BMC Genom. 14, 774 (2013).

86. K. Katoh, D. M. Standley, MAFFT multiple sequence alignment software version 7: Improvements in performance and usability. Mol. Biol. Evol. 30, 772-780 (2013).

87. A. M. Kozlov, D. Darriba, T. Flouri, B. Morel, A. Stamatakis, RAxML-NG: A fast, scalable and user-friendly tool for maximum likelihood phylogenetic inference. Bioinformatics 35, 4453-4455 (2019)

88. M. dos Reis, Z. Yang, Approximate likelihood calculation on a phylogeny for Bayesian estimation of divergence times. Mol. Biol. Evol. 28, 2161-2172 (2011).

89. M. Suyama, D. Torrents, P. Bork, PAL2NAL: Robust conversion of protein sequence alignments into the corresponding codon alignments. Nucleic Acids Res. 34, W609-W612 (2006)

90. S. Kumar, G. Stecher, M. Suleski, S. B. Hedges, TimeTree: A resource for Timelines, Timetrees, and divergence times. Mol. Biol. Evol. 34, 1812-1819 (2017).

91. G. O. Poinar Jr, R. Buckley, Evidence of mycoparasitism and hypermycoparasitism in Early Cretaceous amber. Mycol. Res. 111, 503-506 (2007).

92. C. Cai, R. A. Leschen, D. S. Hibbett, F. Xia, D. Huang, Mycophagous rove beetles highlight diverse mushrooms in the Cretaceous. Nat. Commun. 8, 14894 (2017).

93. L. Guy, J. R. Kultima, S. G. Andersson, genoPlotR: Comparative gene and genome visualization in R. Bioinformatics 26, 2334-2335 (2010).

94. A. M. Bolger, M. Lohse, B. Usadel, Trimmomatic: A flexible trimmer for Illumina sequence data. Bioinformatics 30, 2114-2120 (2014)

95. Y. Liao, G. K. Smyth, W. Shi, featureCounts: An efficient general purpose program for assigning sequence reads to genomic features. Bioinformatics 30, 923-930 (2014).

96. L. W. Meinhardt et al., Genome and secretome analysis of the hemibiotrophic fungal pathogen, Moniliophthora roreri, which causes frosty pod rot disease of cacao: Mechanisms of the biotrophic and necrotrophic phases. BMC Genom. 15, 164 (2014). 